\title{
Eat well to improve smell?
}

\section{ce}

The high-fat

diet reduced

the number

of M72 OSNs

and the

density of their

projections to

the olfactory

bulb

Obesity is linked to cognitive deficits in humans, and consumption of a high-fat diet disrupts neuronal plasticity and function in animals. However, whether high fat intake alters sensory processing is unknown. Fadool and colleagues now show that, in mice, a high-fat diet leads to long-lasting structural and functional changes in the olfactory system.

The activity of olfactory sensory neurons (OSNs) in the main olfactory epithelium is modulated by metabolism-associated molecules such as insulin, suggesting that OSNs

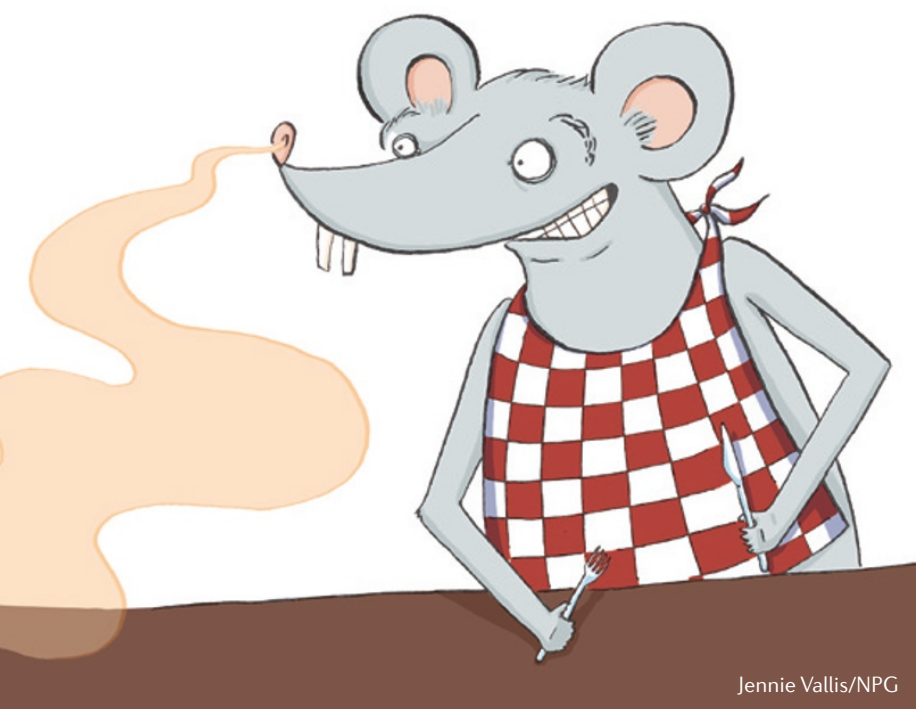

may be affected by dietary changes. To examine this possibility, the authors began feeding mice a high-fat diet at postnatal day 23 and examined the effects on OSNs expressing the M72 olfactory receptor 6 months later.

The high-fat diet reduced the number of M72-expressing OSNs and the density of their projections to the olfactory bulb. By contrast, OSN projections in mice exhibiting genetically induced (rather than diet-induced) obesity as a result of a mutation in melanocortin receptor 4 were normal, confirming that the changes result from dietary fat levels rather than obesity per se. The authors also observed a reduction in the size of the odourevoked electrical response in the main olfactory epithelium, indicating that the altered glomerular architecture affects OSN responsiveness.

To determine the effects of these changes on olfactory-associated behaviour, the authors examined the performance of the mice in a task that required them to learn an association between a particular odorant and a reward (water). Mice fed high-fat diets were initially slower to learn this association than control mice, regardless of the amount of adiposity gained. Furthermore, in a reversal learning paradigm, the mice fed highfat diets were unable to rapidly adapt to a switch in the odorant that was associated with the reward (unlike control mice), demonstrating reduced behavioural or cognitive flexibility.

Can the effects of a high-fat diet be reversed by switching to a control diet? The authors found that, although feeding the mice that had previously been on the high-fat diet a control diet for 5 months significantly reduced their body weight and fasting serum glucose levels, the structural and behavioural deficits were unaltered, indicating that the effects of high fat consumption at early postnatal stages are long-lasting.

This study shows that a high-fat diet can result in persistent changes in the structure and function of the olfactory system. It would be interesting to determine whether these effects are replicated when a high-fat diet begins at different life stages. Given the links between olfaction and food choice, it is possible that these changes might further contribute to the negative effects of a poor diet.

Katherine Whalley

ORIGINAL RESEARCH PAPER Thiebaud, N. et al Hyperlipidemic diet causes loss of olfactory sensory neurons, reduces olfactory discrimination, and disrupts odor-reversal learning.J. Neurosci. 34, 6970-6984 (2014)

FURTHER READING Gómez-Pinilla, F. Brain foods: the effects of nutrients on brain function. Nature Rev. Neurosci. 9, 568-578 (2008) 